\title{
A Remark on the Hull of a Multi-Dimensional Limit-Periodic Potential
}

\author{
Z. Gan* \\ Department of Mathematics, Rice University, Houston, TX 77005, USA
}

\begin{abstract}
We discuss the hull of a multi-dimensional limit-periodic potential and show that such a hull is an inverse limit of product cyclic groups. We present the result in an explicit way, which will be useful for a future study of multi-dimensional limit-periodic Schrödinger operators.
\end{abstract}

Keywords and phrases: limit-periodic potential, inverse limit

Mathematics Subject Classification: 47B36, 47B80, 81Q10

\section{Introduction}

When investigating spectral properties of almost-periodic Schrödinger operators, mathematicians would like to write them as the following:

$$
\left(H_{\omega} u\right)(n)=\sum_{|m-n|=1} u(m)+V_{\omega}(n) u(n)
$$

where

$$
V_{\omega}(n)=f\left(T^{n}(\omega)\right), \quad \omega \in \Omega, n \in \mathbb{Z}^{d}
$$

with a $\mathbb{Z}^{d}$ action by translations $T$ and a continuous sampling function $f: \Omega \rightarrow \mathbb{R}$. If $\Omega=\mathcal{T}^{d}$ (the multiplicative group of all $d$-dimensional complex vectors with entries of norm 1), then $\left\{V_{\omega}(n)\right\}_{n \in \mathbb{Z}^{d}}$ is quasi-periodic and $H_{\omega}$ is called a quasi-periodic Schrödinger operator. If $\Omega$ is a Cantor group with minimal translations, $\left\{V_{\omega}(n)\right\}_{n \in \mathbb{Z}^{d}}$ is limit-periodic and $H_{\omega}$ is called a limit-periodic Schrödinger operator. Conversely, given an almost-periodic Schrödinger operator first, we can just take $\Omega$ as the hull of $\{V(n)\}_{n \in \mathbb{Z}^{d}}$. For a limit-periodic potential $V^{1}$, the hull is a Cantor group with minimal translations. By this way, one can separate base dynamics and sampling function, so that it becomes easy and natural to answer questions of the type how often does phenomenon $X$ occur? [1,3-6] presented many spectral properties of limit-periodic Schrödinger operators under this framework. Because of usefulness of the framework, it becomes necessary to describe group structure of the hull of a limit-periodic potential in detail for a future study of the limit-periodic Schrödinger operators (even though we already know that it is a Cantor group ). [9] studied the hull of a one-dimensional limit-periodic potential, showing that

\footnotetext{
*Corresponding author. E-mail: zheng.gan@rice.edu

Z. G. was supported in part by NSF grant DMS-0800100.

${ }^{1}$ Though $V$ is simply an element of $\ell^{\infty}\left(\mathbb{Z}^{d}\right)$, we call $V$ a potential throughout the paper.
} 
the hull is isomorphic to the inverse limit of a sequence of cyclic groups, that is, a procyclic group. This paper will generalize to the multi-dimensional case and the main result in this paper is Theorem 2.8 , stating that the hull of a multi-dimensional limit-periodic potential is isomorphic to the inverse limit of a sequence of product cyclic groups.

\section{Preliminaries}

Before stating the main result, let us introduce some preliminary facts. It is well known that there is a close connection between the hulls of limit-periodic potentials in $\ell^{\infty}\left(\mathbb{Z}^{d}\right)$ and Cantor groups which admit a minimal $\mathbb{Z}^{d}$ action by translations. For $d=1$, this was worked out in detail in [1, Section 2]; for $d>1$, this was worked out in $[6$, Section 2]. We rewrite some old definitions and results for the reader's convenience.

Definition 2.1. (a) We say that $\Omega$ is a Cantor group if it is an infinite, totally disconnected, metrizable, compact Abelian group. We fix a metric dist on $\Omega$ that is compatible with the topology.

(b) Consider a Cantor group $\Omega$ and a $\mathbb{Z}^{d}$ action by translations, $\left\{T^{n}\right\}_{n \in \mathbb{Z}^{d}}$. That is, there are $\alpha_{1}, \ldots, \alpha_{d} \in \Omega$ such that for $\omega \in \Omega$, we have

$$
T^{n} \omega=\omega+\sum_{j=1}^{d} n_{j} \alpha_{j},
$$

where we write the group operation as.$+^{2}$ We say that the action is minimal if all orbits are dense, that is, for each $\omega \in \Omega$, we have $\overline{\left\{T^{n} \omega: n \in \mathbb{Z}^{d}\right\}}=\Omega$.

Definition 2.2. Let $d \in \mathbb{Z}_{+}$. The group $\mathbb{Z}^{d}$ acts on $\ell^{\infty}\left(\mathbb{Z}^{d}\right)$ as $\left(S_{m} V\right)(n)=V(n-m)$ for $n, m \in \mathbb{Z}^{d}$ and $V \in \ell^{\infty}\left(\mathbb{Z}^{d}\right)$. The set $\operatorname{orb}(V)=\left\{S_{m} V: m \in \mathbb{Z}^{d}\right\}$ is called the orbit of $V$ and the closure of its orbit is called its hull, that is, hull $(V)=\overline{\operatorname{orb}(V)}$. An element $V$ of $\ell^{\infty}\left(\mathbb{Z}^{d}\right)$ is called periodic if its orbit is finite. It is called limit-periodic if it is not periodic and belongs to the closure of the set of periodic elements of $\ell^{\infty}\left(\mathbb{Z}^{d}\right)$.

$V$ is periodic in the sense of Definition 2.2 if and only if it is periodic in each direction, that is, there are $p_{1}, \ldots, p_{d} \in \mathbb{Z}_{+}$such that for all $n=\left(n_{1}, \ldots, n_{d}\right), k=\left(k_{1}, \ldots, k_{d}\right) \in \mathbb{Z}^{d}$, we have $V\left(n_{1}+k_{1} p_{1}, \ldots, n_{d}+\right.$ $\left.k_{d} p_{d}\right)=V\left(n_{1}, \ldots, n_{d}\right)$. We will call $p=\left(p_{1}, \ldots, p_{d}\right) \in\left(\mathbb{Z}_{+}\right)^{d}$ a periodicity vector of $V$. There exists a smallest periodicity vector for a periodic $V$. The following proposition describes how limit-periodic potentials in $\ell^{\infty}\left(\mathbb{Z}^{d}\right)$ may be generated.

Proposition 2.3. Suppose $\Omega$ is a Cantor group that admits a minimal $\mathbb{Z}^{d}$ action by translations, $\left\{T^{n}\right\}_{n \in \mathbb{Z}^{d}}$. Then, for every $f \in C(\Omega, \mathbb{R})$ and every $\omega \in \Omega$, the potential $V_{\omega}$ of $\ell^{\infty}\left(\mathbb{Z}^{d}\right)$ defined by $V_{\omega}(n)=f\left(T^{n} \omega\right)$ is limit-periodic. Moreover, for each $\omega \in \Omega$, we have hull $\left(V_{\omega}\right)=\left\{V_{\tilde{\omega}}: \tilde{\omega} \in \Omega\right\}$.

We first prove the following simple lemma:

Lemma 2.4. Suppose that $\left\{T^{n}\right\}_{n \in \mathbb{Z}^{d}}$ is an action by translations as in (2.1) on the compact Abelian group $\Omega$. Then, for each $j \in\{1, \ldots, d\}$, there is a sequence $\left\{n_{k}^{(j)}\right\}_{k \in \mathbb{Z}_{+}} \subset \mathbb{Z}_{+}$such that $\lim _{k \rightarrow \infty} n_{k}^{(j)} \alpha_{j}=\omega_{e}$, the identity element of $\Omega$.

Proof. Let us fix $j$ and explain how to find $\left\{n_{k}^{(j)}\right\}_{k \in \mathbb{Z}_{+}} \subset \mathbb{Z}_{+}$. Since $\Omega$ is compact, there exists an increasing sequence of positive integers $m_{k} \rightarrow \infty$ such that $m_{k} \alpha_{j}$ converges to some $\omega \in \Omega$ as $k \rightarrow \infty$. For each $k$, choose $\tilde{m}_{k} \in\left\{m_{k+\ell}: \ell \geq 1\right\}$ such that $n_{k}^{(j)}:=\tilde{m}_{k}-m_{k} \geq k$. Then, $n_{k}^{(j)} \rightarrow \infty$ as $k \rightarrow \infty$ and $\lim _{k \rightarrow \infty} n_{k}^{(j)} \alpha_{j}=\omega-\omega=\omega_{e}$, as desired.

\footnotetext{
${ }^{2}$ While + is a natural way to denote the group operation in the abstract setting, for the concrete groups that arise as hulls of limit-periodic elements of $\ell^{\infty}\left(\mathbb{Z}^{d}\right)$, this is ambiguous. Thus, in the concrete setting, we will prefer to use $\cdot$ to denote the group operation.
} 
Proof of Proposition 2.3. For a given $\varepsilon>0$, we may choose a compact open neighborhood $U$ of the identity $\omega_{e} \in \Omega$ that is small enough so that $\left|f\left(\omega+\omega_{U}\right)-f(\omega)\right|<\varepsilon$ for every $\omega_{U} \in U$ and every $\omega \in \Omega$.

Since $U$ is compact and open, we can choose $\delta>0$ such that $\operatorname{dist}\left(\omega_{U}, \omega_{\Omega \backslash U}\right)>\delta$ for every $\omega_{U} \in U$ and every $\omega_{\Omega \backslash U} \in \Omega \backslash U$.

Lemma 2.4 shows that we can choose $p_{1}, \ldots, p_{d} \in \mathbb{Z}_{+}$such that $d\left(\omega_{e}, p_{j} \alpha_{j}\right)<\delta$ for $j=1, \ldots, d$. By the defining property of $\delta$, it follows that the closure of

$$
\left\{\sum_{j=1}^{d} n_{j} p_{j} \alpha_{j}: n=\left(n_{1}, \ldots, n_{d}\right) \in \mathbb{Z}^{d}\right\}
$$

is a compact subgroup of $\Omega$ that is contained in $U$. Its index is bounded by $\prod p_{j}$.

Now, given $f \in C(\Omega, \mathbb{R})$ and $\omega \in \Omega$, we consider the potential $V_{\omega}$ of $\ell^{\infty}\left(\mathbb{Z}^{d}\right)$ defined by $V_{\omega}(n)=f\left(T^{n} \omega\right)$. With the arbitrary choice of $\varepsilon>0$ above and the resulting $U$ and $\delta>0$, we consider the following potential $V_{\omega}^{p}$ of $\ell^{\infty}\left(\mathbb{Z}^{d}\right), V_{\omega}^{p}(n)=f\left(T^{\tilde{n}} \omega\right)$, where $\tilde{n}=\left(\tilde{n}_{1}, \ldots, \tilde{n}_{d}\right)$ is defined by $\tilde{n}_{j} \in\left\{0, \ldots, p_{j}-1\right\}$ and $\tilde{n}_{j} \equiv n_{j}$ $\bmod p_{j}$. Thus, $V_{\omega}^{p}$ is periodic. We have

$$
\begin{aligned}
\left\|V_{\omega}-V_{\omega}^{p}\right\|_{\infty} & =\sup _{n \in \mathbb{Z}^{d}}\left|V_{\omega}(n)-V_{\omega}^{p}(n)\right| \\
& =\sup _{n \in \mathbb{Z}^{d}}\left|f\left(T^{n} \omega\right)-f\left(T^{\tilde{n}} \omega\right)\right| \\
& =\sup _{n \in \mathbb{Z}^{d}}\left|f\left(T^{\tilde{n}} \omega+\left(T^{n} \omega-T^{\tilde{n}} \omega\right)\right)-f\left(T^{\tilde{n}} \omega\right)\right| \\
& <\varepsilon .
\end{aligned}
$$

The first three steps follow by simple rewriting, and the final step follows from the choice of $U$ and the fact that, by construction, $T^{n} \omega-T^{\tilde{n}} \omega$ belongs to $U$. This shows that $V_{\omega}$ is limit-periodic since $\varepsilon>0$ is arbitrary and $V_{\omega}^{p}$ is periodic.

The statement hull $\left(V_{\omega}\right)=\left\{V_{\tilde{\omega}}: \tilde{\omega} \in \Omega\right\}$ follows since both sides are compact and contain $\operatorname{orb}\left(V_{\omega}\right)$ as a dense subset (for the right-hand side, this is a consequence of the minimality of the action). This completes the proof of the proposition.

Thus, we have seen that a Cantor group that admits a minimal $\mathbb{Z}^{d}$ action by translations and a continuous sampling function give rise to limit-periodic potentials of $\ell^{2}\left(\mathbb{Z}^{d}\right)$. Let us now turn to the converse. That is, given a limit-periodic potential of $\ell^{2}\left(\mathbb{Z}^{d}\right)$, we want to show that it arises in this way.

Proposition 2.5. Suppose $V \in \ell^{\infty}\left(\mathbb{Z}^{d}\right)$ is limit-periodic. Then, hull $(V)$ is compact and it has a unique topological group structure so that $V$ is the identity element and $\mathbb{Z}^{d} \rightarrow \operatorname{hull}(V), m \mapsto S_{m} V$ is a homomorphism. Moreover, the group structure is Abelian and there exist arbitrarily small compact open neighborhoods of $V$ in hull $(V)$ that are finite index subgroups, and hull $(V)$ admits a minimal $\mathbb{Z}^{d}$ action by translations.

Proof. Since $V$ is limit-periodic, we can find for each $\varepsilon>0$, a periodic $V_{p}$ with $\left\|V-V_{p}\right\|_{\infty}<\varepsilon$. Since $\operatorname{orb}\left(V_{p}\right)$ is finite, it follows that $\operatorname{orb}(V)$ is contained in the $\varepsilon$-neighborhood of a finite set. That is, $\operatorname{orb}(V)$ is totally bounded and hence its closure hull $(V)$ is compact.

Obviously, there is a unique group structure on $\operatorname{orb}(V)$ such that $\mathbb{Z}^{d} \rightarrow \operatorname{orb}(V), m \mapsto S_{m} V$ is a homomorphism. Our goal is to show that it extends uniquely to a group structure on hull $(V)$. It suffices to show uniform continuity of the group structure on $\operatorname{orb}(V)$. This will then also show that the resulting extension of the group structure to hull $(V)$ is Abelian. We have

$$
\begin{aligned}
\left\|S_{m_{1}+k_{1}} V-S_{m_{2}+k_{2}} V\right\|_{\infty} & =\left\|S_{m_{1}-m_{2}} V-S_{k_{2}-k_{1}} V\right\|_{\infty} \\
& \leq\left\|S_{m_{1}-m_{2}} V-V\right\|_{\infty}+\left\|V-S_{k_{2}-k_{1}} V\right\|_{\infty} \\
& =\left\|S_{m_{1}} V-S_{m_{2}} V\right\|_{\infty}+\left\|S_{k_{1}} V-S_{k_{2}} V\right\|_{\infty} .
\end{aligned}
$$


Here, the first and the third step follow since translations are isometries and the second step follows from the triangle inequality. Put differently, if $a, b, c, d \in \operatorname{orb}(V)$ and we denote the group operation by $\cdot$, then $\|a \cdot b-c \cdot d\|_{\infty} \leq\|a-c\|_{\infty}+\|b-d\|_{\infty}$, which shows the desired uniform continuity.

To prove the last statement about finite index subgroups in small neighborhoods of the identity, let $\varepsilon>0$ be given. Choose a periodic $V_{p} \in \ell^{\infty}\left(\mathbb{Z}^{d}\right)$ with $\left\|V-V_{p}\right\|_{\infty}<\frac{\varepsilon}{2}$. Also, there are $p_{1}, \ldots, p_{d} \in \mathbb{Z}_{+}$such that for all $n=\left(n_{1}, \ldots, n_{d}\right), k=\left(k_{1}, \ldots, k_{d}\right) \in \mathbb{Z}^{d}$, we have $V_{p}\left(n_{1}+k_{1} p_{1}, \ldots, n_{d}+k_{d} p_{d}\right)=V_{p}\left(n_{1}, \ldots, n_{d}\right)$. In other words, $V_{p}$ is invariant under $S_{m}$ for every $m \in\left(p_{1} \mathbb{Z}\right) \times \cdots \times\left(p_{d} \mathbb{Z}\right)$. Clearly, the closure of $\left\{S_{m} V: m \in\left(p_{1} \mathbb{Z}\right) \times \cdots \times\left(p_{d} \mathbb{Z}\right)\right\}$, which we denote by $\operatorname{hull}_{p}(V)$, is a compact subgroup of hull $(V)$ of index at most $\prod p_{j}$. Since hull $(V)$ is the union of finitely many disjoint translates of $\operatorname{hull}_{p}(V)$, it follows that $\operatorname{hull}_{p}(V)$ is also open. By the invariance property of $V_{p}$, hull $p(V)$ is contained in the $\frac{\varepsilon}{2}$-ball around $V_{p}$, and hence it is contained in the $\varepsilon$-ball around $V$.

Last, the minimal $\mathbb{Z}^{d}$ action is given by $T^{n}=S_{n}$ with the translations $S_{n}$ introduced above. Note that this action is indeed an action by translations in the sense of Definition 2.1, simply choosing $\alpha_{j}=$ $T^{(0, \cdots, 1, \cdots, 0)}(V)$ with the $j$-th component being 1 . Let us show that this action is minimal. It suffices to show that for $\omega_{1}, \omega_{2} \in \operatorname{hull}(V)$ and $\varepsilon>0$, there is $n \in \mathbb{Z}^{d}$ such that $\operatorname{dist}\left(T^{n} \omega_{1}, \omega_{2}\right)=\left\|T^{n} \omega_{1}-\omega_{2}\right\|_{\infty}<\varepsilon$. We can choose $n_{1}, n_{2} \in \mathbb{Z}^{d}$ such that $\left\|\omega_{j}-T^{n_{j}} V\right\|_{\infty}<\frac{\varepsilon}{2}, j=1,2$. Now set $n:=n_{2}-n_{1}$. Putting everything together and using that $T$ is an isometry, we find

$$
\begin{aligned}
\left\|T^{n} \omega_{1}-\omega_{2}\right\|_{\infty} & \leq\left\|T^{n} \omega_{1}-T^{n+n_{1}} V\right\|_{\infty}+\left\|T^{n+n_{1}} V-\omega_{2}\right\|_{\infty} \\
& =\left\|\omega_{1}-T^{n_{1}} V\right\|_{\infty}+\left\|T^{n_{2}} V-\omega_{2}\right\|_{\infty} \\
& <\varepsilon .
\end{aligned}
$$

Next we will rewrite some results from [2, Appendix 1] in the $d$-dimensional context, introducing the frequency module for $\operatorname{hull}(V)$. Denote $\operatorname{hull}(V)$ by $\Omega_{V}$. $\hat{\Omega}_{V}$, of characters on $\Omega_{V}$, is naturally a topological subgroup of $\mathcal{T}^{d}$. By taking inverse image from $[0,1]^{d}$ to $\mathcal{T}^{d}$ under the map $\left(\alpha_{1}, \cdots, \alpha_{d}\right) \rightarrow$ $\left(e^{2 i \pi \alpha_{1}}, \cdots, e^{2 i \pi \alpha_{d}}\right)$, we obtain $\hat{\Omega}_{V}$ as a subgroup of $[0,1]^{d}$, called the frequency module of $V$. $\hat{\Omega}_{V}$ is countable since $\Omega_{V}$ has a countable dense set. Since $\hat{\Omega}_{V}$ is a subgroup of $[0,1]^{d}$, given $\alpha, \beta \in \hat{\Omega}_{V}$, and integers $n_{1}, n_{2}$, we have that $n_{1} \alpha+n_{2} \beta \bmod \mathbf{1} \in \hat{\Omega}_{V}$, i.e. $\hat{\Omega}_{V}$ is a module over $\mathbb{Z}$ (one should automatically consider $\bmod \mathbf{1}$ when discussing $\hat{\Omega}_{V}$ so that we don't have to write $\bmod \mathbf{1}$ every time).

The Peter-Weyl theorem assures us that any $V$ is a uniform limit of finite sums of the form $\sum_{j=1}^{k} c_{j} e^{i 2 \pi \alpha^{(j)} \cdot n}$ with $\alpha^{(j)} \in \hat{\Omega}_{V}$. From this it follows that

Proposition 2.6. The frequency module, $\hat{\Omega}_{V}$, is the module generated by

$$
\left\{\alpha: \lim _{k \rightarrow \infty} \frac{1}{(2 k)^{d}} \sum_{n \in[-k, k]^{d}} V(n) e^{-i 2 \pi n \cdot \alpha} \neq 0, \alpha \in[0,1]^{d}\right\} .
$$

For $\alpha, \beta \in[0,1]^{d}$, we say that they have a common divisor $\gamma \in[0,1]^{d}$ if there exist $n, m \in \mathbb{Z}^{d}$ such that for $1 \leq j \leq d, n_{j} \gamma_{j}=\alpha_{j}$ and $m_{j} \gamma_{j}=\beta_{j}$ respectively. Like [2, Theorem A.1.3], we then have

Proposition 2.7. $V$ is limit-periodic if and only if $\hat{\Omega}_{V}$ has the property that any $\alpha, \beta \in \hat{\Omega}_{V}$ have a common divisor in $\hat{\Omega}_{V}$.

Proof. For $\alpha, \beta \in \hat{\Omega}_{V}$, by Proposition 2.6

$$
a=\lim _{k \rightarrow \infty} \frac{1}{(2 k)^{d}} \sum_{n \in[-k, k]^{d}} V(n) e^{-i 2 \pi n \cdot \alpha}
$$

and

$$
b=\lim _{k \rightarrow \infty} \frac{1}{(2 k)^{d}} \sum_{n \in[-k, k]^{d}} V(n) e^{-i 2 \pi n \cdot \beta}
$$


are both non-zero. Choose a periodic potential $P \in \ell^{\infty} \mathbb{Z}$ with

$$
\|P-V\|_{\infty} \leq \frac{1}{2} \min (|a|,|b|) .
$$

It follows that

$$
\lim _{k \rightarrow \infty} \frac{1}{(2 k)^{d}} \sum_{n \in[-k, k]^{d}} P(n) e^{-i 2 \pi n \cdot \gamma} \neq 0
$$

for $\gamma=\alpha, \beta$. Let $p=\left(p_{1}, \cdots, p_{2}\right)$ be the smallest periodicity vector of $P$. The frequency module of $P$ can be generated by $\left\{\left(0, \cdots, 0,1 / p_{j}, 0, \cdots, 0\right): 1 \leq j \leq d\right\}$ as a $\mathbb{Z}$ module. Since $\alpha, \beta$ both belong to the frequency module of $P, 1 / p_{j}$ must divide both $\alpha_{j}$ and $\beta_{j}$. So $\left(1 / p_{1}, \cdots, 1 / p_{d}\right)$ is a common divisor of $\alpha$ and $\beta$. Similarly any finite subset of the generating set $\left\{\alpha: \lim _{k \rightarrow \infty} \frac{1}{(2 k)^{d}} \sum_{n \in[-k, k]^{d}} V(k) e^{-i 2 \pi n \cdot \alpha} \neq 0\right\}$ have a common divisor. Since we can select the greatest common divisor, the property follows.

Conversely, if $\hat{\Omega}_{V}$ has the property, any finite $\operatorname{sum} \sum_{j=1}^{k} c_{j} e^{i 2 \pi \alpha^{(j)} \cdot n}$ with $\alpha_{j} \in \hat{\Omega}_{V}$ is periodic since the $\alpha^{(j)}$ have a common divisor. By the Peter-Weyl theorem, $V$ is limit-periodic.

Since any finite collection of $\alpha^{(j)}$ have a common divisor in $\hat{\Omega}_{V}$ and furthermore $V$ is a uniform limit of finite sums of the form $\sum_{j=1}^{k} c_{j} e^{i 2 \pi \alpha^{(j)} \cdot n}$, we can find a sequence of periodic $P^{(k)} \in \ell^{\infty}\left(\mathbb{Z}^{d}\right)$ which satisfy the following: (i) $\lim _{k \rightarrow \infty} P^{(k)}=V$ in $\ell^{\infty}$-norm. (ii) Write $p^{(k)}$ as the smallest periodicity vector of $P^{(k)}$. We have $p_{i}^{(k)} \mid p_{i}^{(k+1)}$ for every $1 \leq i \leq d$. (iii) $\left(\frac{1}{p_{1}^{(k)}}, \cdots, \frac{1}{p_{d}^{(k)}}\right) \in \hat{\Omega}_{V}$ for all $k$.

Write $G_{V}=\left\{\left(\frac{1}{p_{1}^{(k)}}, \cdots, \frac{1}{p_{d}^{(k)}}\right): k \in \mathbb{Z}_{+}\right\}$. Clearly, $G_{V} \subset \hat{\Omega}_{V}$. From the proof of Proposition 2.7, we can see that for any vector $n \in \mathbb{Z}^{d}$ and $\alpha \in G_{V},\left(n_{1} \alpha_{1}, n_{2} \alpha_{2}, \cdots, n_{d} \alpha_{d}\right) \in \hat{\Omega}_{V}$. Moreover, with the common divisor property, $\hat{\Omega}_{V}$ can be generated by $G_{V}$ by such a $\mathbb{Z}^{d}$ action, i.e., entry by entry multiplication. Write $F_{V}=\left\{\left(p_{1}^{(k)}, \cdots, p_{d}^{(k)}\right): k \in \mathbb{Z}_{+}\right\}$. We call $F_{V}$ a frequency integer vector set of $V$. Write $X^{(k)}=\mathbb{Z}_{p_{1}^{(k)}} \times \cdots \times \mathbb{Z}_{p_{d}^{(k)}}$, a product cyclic group. Let $X$ be the inverse limit of $\left\{X^{(k)}\right\}_{k \in \mathbb{Z}_{+}}$(we will introduce the inverse limit concept in the next section). Our main result is the following.

Theorem 2.8. $\Omega_{V}$ is isomorphic to $X$.

\section{Inverse limits}

A directed set is a partially ordered set $I$ such that for all $i_{1}, i_{2} \in I$ there is an element $j \in I$ for which $i_{1} \leq j$ and $i_{2} \leq j$.

Definition 3.1. An inverse system $\left(X_{i}, \phi_{i j}\right)$ of topological groups indexed by a directed set $I$ consists of a family $\left(X_{i} \mid i \in I\right)$ of topological groups and a family $\left(\phi_{i j}: X_{j} \rightarrow X_{i} \mid i, j \in I, i \leq j\right)$ of continuous homomorphisms such that $\phi_{i i}$ is the identity map $\operatorname{id}_{X_{i}}$ for each $i$ and $\phi_{i j} \phi_{j k}=\phi_{i k}$ whenever $i \leq j \leq k$.

Definition 3.2. An inverse limit $\left(X, \phi_{i}\right)$ of an inverse system $\left(X_{i}, \phi_{i j}\right)$ of topological groups is a topological group together with a compatible family $\left(\phi_{i}: X \rightarrow X_{i}\right)$ of continuous homomorphisms with the following universal property: whenever $\left(\varphi_{i}: Y \rightarrow X_{i}\right)$ is a compatible family of continuous homomorphisms from a topological group $Y$, there is a unique continuous homomorphism $\varphi: Y \rightarrow X$ such that $\phi_{i} \varphi=\varphi_{i}$ for each $i$.

Proposition 3.3. [10, Proposition 1.1.4] Let $\left(X_{i}, \phi_{i j}\right)$ be an inverse system of topological groups, indexed by $I$.

(1). There exists an inverse limit $\left(X, \phi_{i}\right)$ of $\left(X_{i}, \phi_{i j}\right)$, for which $X$ is a topological group and the maps $\phi_{i}$ are continuous homomorphisms. 
(2). If $\left(X^{(1)}, \phi_{i}^{(1)}\right)$ and $\left(X^{(2)}, \phi_{i}^{(2)}\right)$ are inverse limits of the inverse system, then there is an isomorphism $\bar{\phi}: X^{(1)} \rightarrow X^{(2)}$ such that $\phi_{i}^{(2)} \bar{\phi}=\phi_{i}^{(1)}$ for each $i$.

(3). Write $G=\prod_{i \in I} X_{i}$ with the product topology and for each $i$ write $\pi_{i}$ for the projection map from $G$ to $X_{i}$. Define

$$
X=\left\{c \in G: \phi_{i j} \pi_{j}(c)=\pi_{i}(c) \text { for all } i, j \text { with } j \geq i\right\}
$$

and $\phi_{i}=\left.\pi_{i}\right|_{X}$ for each $i$. Then $\left(X, \phi_{i}\right)$ is an inverse limit of $\left(X_{i}, \phi_{i j}\right)$.

The above proposition shows that the inverse limit of an inverse system $\left(X_{i}, \phi_{i j}\right)$ exists and is unique up to isomorphism. A topological profinite group is by definition an inverse limit of finite topological groups. The inverse limit of a sequence of cyclic groups is called a procyclic group.

\section{Proof of Theorem 2.8}

For a $d$-dimensional limit-periodic potential $V$, there exist periodic potentials $\left\{P^{(k)}\right\}_{k \in \mathbb{Z}_{+}}$converging to $V$ uniformly. $P^{(k)}$ has the smallest periodicity vector $p^{(k)}=\left(p_{1}^{(k)}, \cdots, p_{d}^{(k)}\right)$. Let's consider hull $(V)$. $\operatorname{hull}(V)$ has a strongly minimal $\mathbb{Z}^{d}$ action $T^{n}=S_{n}$ with $\alpha_{j}=T^{(0, \cdots, 1, \cdots, 0)}(V)$ (the $j$-th component being 1). $F_{V}=\left\{\left(p_{1}^{(k)}, \cdots, p_{d}^{(k)}\right): k \in \mathbb{Z}_{+}\right\}$is a frequency integer vector set of $\operatorname{hull}(V)$.

From $(0, \cdots, 0)$ to $\left(p_{1}^{(k)}-1, \cdots, p_{d}^{(k)}-1\right)$, there are $h^{(k)}=p_{1}^{(k)} p_{2}^{(k)} \cdots p_{d}^{(k)}$ vectors. Denote these vectors by $\gamma^{(k 1)}, \gamma^{(k 2)}, \cdots, \gamma^{\left(k h^{(k)}\right)}$ for writing convenience. Make $\gamma^{(11)}=(0, \cdots, 0)$ and furthermore $\gamma^{(k i)}=\gamma^{(m i)}$ when $k>m$ with neat ordering. $\gamma_{j}^{(k 1)}$ means the $j$-th entry of the vector $\gamma^{(k 1)}$. Write

$$
H^{(k i)}=\left\{\sum_{j=1}^{d}\left(\gamma_{j}^{(k i)}+h_{j} p_{j}^{(k)}\right) \alpha_{j}: h_{j} \in \mathbb{Z}\right\}
$$

and

$$
U^{(k i)}=\overline{\left\{\sum_{j=1}^{d}\left(\gamma_{j}^{(k i)}+h_{j} p_{j}^{(k)}\right) \alpha_{j}: h_{j} \in \mathbb{Z}\right\}}
$$

in the space hull $(V)$ for $1 \leq i \leq h^{(k)}$. Next we will first show that

$$
\bigcap_{k=m}^{\infty} U^{(k i)}=\left\{\sum_{j=1}^{d} \gamma_{j}^{(m i)} \alpha_{j}\right\}
$$

for $1 \leq i \leq h^{(m)}$.

Assume $j=1$. Like in the proof of Proposition 2.3, given any $E$, a compact open neighborhood of $V$, we can choose $\delta>0$ such that $\operatorname{dist}\left(\omega_{E}, \omega_{\text {hull }(V) \backslash E}\right)>\delta$ for every $\omega_{E} \in E$ and every $\omega_{\text {hull }(V) \backslash E} \in \operatorname{hull}(V) \backslash E$. Choose $P^{(m)}$ so that $\left\|V-P^{(m)}\right\| \leq \delta / 2$. We have $\left\|V-T^{\left(0, \cdots, p_{j}^{(m)}, \cdots, 0\right)}(V)\right\|=$ $\left\|V-P^{(m)}+T^{\left(0, \cdots, p_{j}^{(m)}, \cdots, 0\right)}\left(P^{(m)}\right)-T^{\left(0, \cdots, p_{j}^{(m)}, \cdots, 0\right)}(V)\right\| \leq \delta$ for $j=1,2, \cdots, d$. By the defining property of $\delta$, it follows that $p_{j}^{(m)} \alpha_{j}$ is contained in $E$. Then $U^{(m 1)}$ is contained in $E$. So we can conclude that $\bigcap_{k=1}^{\infty} U^{(k 1)}=\{V\}=\left\{\sum_{j=1}^{d} \gamma_{j}^{(11)} \alpha_{j}\right\}$. Note that for $1 \leq i \leq h^{(m)}, \gamma^{(k i)}=\gamma^{(m i)}$ when $k \geq m$ and $U^{(k+1) i} \subset U^{(k i)}$ since $p_{i}^{(k)} \mid p_{i}^{(k+1)}$. Similarly, we have

$$
\bigcap_{k=m}^{\infty} U^{(k i)}=\left\{\sum_{j=1}^{d} \gamma_{j}^{(m i)} \alpha_{j}\right\} .
$$


Denote $\operatorname{hull}(V) / U^{(k 1)}$ by $X^{(k)}$. Let $\phi_{i j}: X^{(j)} \rightarrow X^{(i)}$ by $\phi_{i j}\left(\omega U^{(j 1)}\right)=\omega U^{(i 1)}$ for $\omega \in \operatorname{hull}(V)$ and $j>i$. Then $\left\{X^{(j)}, \phi_{i j}\right\}$ is an inverse system, and we write the inverse limit as $X$. We will show that $\operatorname{hull}(V) \cong X$. Let $\psi: \operatorname{hull}(V) \rightarrow X$ be the continuous mapping induced by the canonical continuous surjection $\psi_{k}: \operatorname{hull}(V) \rightarrow X^{(k)}$. It is easy to see that $\psi$ is a continuous surjection. To prove that $\psi$ is a homeomorphism, it suffices then to prove that it is an injection, since $\operatorname{hull}(V)$ is compact. Let $\omega_{1}, \omega_{2} \in \operatorname{hull}(V)$. There exists a open neighborhood $O$ of $\omega_{1}$ that excludes $\omega_{2}$. Choose $k$ large enough so that for any $\omega \in U^{(k 1)}$ we have $\omega \omega_{1} \in O$ (this is because $U^{(k 1)}$ is a sufficiently small neighborhood of $V$ ). Then $\omega_{2} \notin \omega_{1} U^{(k 1)}$, and $\psi_{k}\left(\omega_{1}\right) \neq \omega_{2} U^{(k 1)}$. So, $\psi\left(\omega_{1}\right) \neq \psi\left(\omega_{2}\right)$. Thus, $\psi$ is an injection.

The only thing left is to show $\operatorname{hull}(V) / U^{(k 1)} \cong Z_{p_{1}^{(k)}} \times Z_{p_{2}^{(k)}} \times \cdots \times Z_{p_{d}^{(k)}}$, where $Z_{p_{j}^{(k)}}$ is the $p_{j}^{(k)}$ cyclic group (note that we only need to consider a large $k$ ). Clearly, $\operatorname{orb}(V)=\bigcup_{i=1}^{h^{(k)}} H^{(k i)}$. So we have $\operatorname{hull}(V)=\bigcup_{i=1}^{h^{(k)}} U^{(k i)}$. By (4.1) we have $U^{(k i)} \bigcap U^{(k j)}=\emptyset$ when $k$ is large enough since hull $(V)$ is totally disconnected. So we have

$$
\operatorname{hull}(V) / U^{(k 1)}=\left\{\left[\sum_{j=1}^{d} \gamma_{j}^{(k 1)} \alpha_{j}\right],\left[\sum_{j=1}^{d} \gamma_{j}^{(k 2)} \alpha_{j}\right], \cdots,\left[\sum_{j=1}^{d} \gamma_{j}^{\left(k h^{(k)}\right)} \alpha_{j}\right]\right\},
$$

which has $h^{(k)}$ elements. We can map hull $(V) / U^{(k 1)}$ to $Z_{p_{1}^{(k)}} \times Z_{p_{2}^{(k)}} \times \cdots \times Z_{p_{d}^{(k)}}$ by $\left[\sum_{j=1}^{d} \gamma_{j}^{(k i)} \alpha_{j}\right] \rightarrow$ $\left(\gamma_{1}^{(k i)}, \gamma_{2}^{(k i)}, \cdots, \gamma_{d}^{(k i)}\right)$. It is easy to see that such a map is an isomorphic map. The proof is done.

Acknowledgements. I am deeply grateful to David Damanik, who encouraged me to write this paper. His comments have been very helpful for completion of the paper.

\section{References}

[1] A. Avila, On the spectrum and Lyapunov exponent of limit periodic Schrödinger operators, Commun. Math. Phys. 288 (2009), 907-918

[2] J. Avron, B. Simon, Almost periodic Schrödinger operators. I. Limit periodic potentials, Commun. Math. Phys. 82 (1981), 101-120

[3] D. Damanik, Z. Gan, Spectral properties of limit-periodic Schrödinger operators, Commun. Pure Appl. Anal. 10 (2011), 859-871

[4] D. Damanik, Z. Gan, Limit-periodic Schrödinger operators in the regime of positive Lyapunov exponents, J. Funct. Anal. 258 (2010), 4010-4025

[5] D. Damanik, Z. Gan, Limit-periodic Schröinger operators with uniformly localized eigenfunctions, J. d'Analyse Math, 115 (2011), 33-49

[6] D. Damanik, Z. Gan, Limit-Periodic Schrödinger Operators on $\mathbb{Z}^{d}$ : Uniform Localization, preprint

[7] R. del Rio, S. Jitomirskaya, Y. Last, B. Simon, What is localization?, Phys. Rev. Lett. 75 (1995), 117-119

[8] R. del Rio, S. Jitomirskaya, Y. Last, B. Simon, Operators with singular continuous spectrum, IV. Hausdorff dimensions, rank one perturbations, and localization, J. Anal. Math. 145 (1997), 312-322

[9] Z. Gan, An exposition of the connection between limit-periodic potentials and profinite groups, Math. Model. Nat. Phenom. 5:4 (2010), 158-174

[10] J. Wilson. Profinite Groups, Oxford University Press, New York, USA, 1998 